\section{A new infective endocarditis} information card

\section{A credit card-sized information card has} been created to simplify communication between the cardiologist and the dentist and to clarify the wishes of the patient.

NICE guidance has changed recently to recognise that antibiotic prophylaxis may sometimes be indicated in a high-risk patient having invasive dental procedures. ${ }^{1}$ Furthermore, as a result of changes in the law on patient consent, ${ }^{2}$ new Scottish Dental Clinical Effectiveness Programme advice on implementing the NICE guidelines, ${ }^{3}$ which have been adopted nationally, stress the need for the patient to discuss with their cardiologist or surgeon whether or not antibiotic prophylaxis is indicated and to record the decision. The card (pictured) allows this discussion to be recorded and kept by the patient. This will make it a lot easier for dentists to confirm that this discussion has happened and if both patient and cardiologist wish the patient to receive antibiotic prophylaxis.

This card has been endorsed by the British Heart Valve Society and the British Cardiovascular Society. The card also gives guidance on what cardiac conditions are regarded as moderate or high-risk for

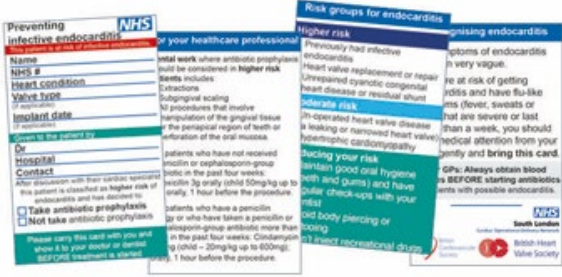

endocarditis, what dental procedures are high-risk and what antibiotics should be prescribed.

Early endocarditis diagnosis improves outcomes, but diagnosis can be difficult because the early signs may be indistinguishable from self-limiting viral illnesses. It is important, therefore, that dentists and any of their patients at increased risk of endocarditis, whether or not they take antibiotic prophylaxis, know what signs and symptoms to look for and what action to take. ${ }^{2}$

\section{References}

1. NICE. Prophylaxis against infective endocarditis: antimicrobial prophylaxis against infective endoacrditis in adults and children undergoing interventional procedures. 2016. Available at: https://www.nice.org uk/guidance/cg64 (accessed 13 March 2019).

2. Thornhill M H, Dayer M, Lockhart P B et al. Guidelines on prophylaxis to prevent infective endocarditis. Br Dent J 2016; 220: 51-56

3. Scottish Dental Clinical Effectiveness Programme. Antibiotic prophylaxis against infective endocarditis. 2018. Available at: http://www.sdcep.org.uk/published-guidance/antibiotic-prophylaxis (accessed 13 March 2019).

\title{
Families encouraged to brush teeth together
}

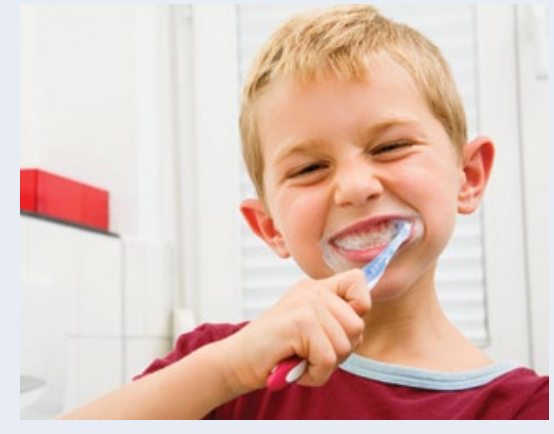

Simplyhealth Professionals has launched its annual Big Family Brush-Up campaign, which runs from 24 June to 31 August 2019, to help families to brush together and equip parents with practical tips and tools to engage their children in establishing a proper brushing routine.

Now in its fifth year, the Big Family Brush-Up challenges families to really focus on their brushing habits for a four-week period over the summer to ensure children are brushing thoroughly for two minutes, twice a day. To track and reward their progress, Simplyhealth Professionals are encouraging children to fill in a brushing chart every morning and night for four weeks, which they can download from the campaign microsite. At the end of the challenge, it's hoped that families will have established a good oral health routine that they can continue into the future.

Families can visit www.bigfamilybrushup. co.uk to find a wealth of oral health information including Simplyhealth's Little Book of Healthy Smiles booklet which provides dental advice and brushing tips from other parents; an animated step-by-step brushing video to inspire young brushers; a downloadable brushing chart, and lots of blogs and social media competitions.

\section{Rotting meat removed from dinosaur's teeth}

A finalist in the 10-13 age group in BBC Radio 2's

'500 Words' competition, written by Millie Robinson, tells the story of 'TyrannosOral Hygiene' (https://bbc. in $/ 2 X 7$ tuxB).

'500 Words' is a short story-writing competition for children between the ages of five and 13, launched in 2011 by Chris Evans. In 2018, 134,790 children entered the competition, and since it launched, the competition has received over 800,000 short stories.

'Tyrannos-Oral Hygiene' was broadcast on 14 June 2019, read by actor Michael Sheen. It tells the story of a 12-foot Tyrannosaurus Rex called Tyrone King who is afraid of the dentist, but overcomes his fear as he has toothache, and goes to see a Dr Gummeridge.

As Tyrone is unable to fit through the dental practice door, Dr Gummeridge examines him in the car park, and when he opens his massive jaw, she almost faints as 'his breath smelt of mouldy cheese and socks but ten times worse!' The dentist also notes that even from a distance, she can see that Tyrone's 'banana-size' teeth were black.

Tyrone mumbles that he has not seen a dentist for around 65 million years, and deciding to tackle the task of cleaning Tyrone's teeth, Dr Gummeridge assembles 'four gardeners, three builders, two plasterers and the fire brigade from the local village' to help her.

Young author Millie describes delightfully how 'the builders used a cement mixer to mix the filling for all the nasty cavities' and 'the plasterers filled the holes and smoothed the mixture down'. In the end, Tyrone is a happy dental patient and departs with a wink and a smile to show off his sparkly smile to his 'dino friends'.

To read the full short story and other entries to the competition, visit https:// bbc.in/2X7tuxB. 